\title{
INDEX OF AUTHORS
}

Page numbers in italics refer to abstracts of papers given at a meeting of the Genetical Society and to titles of books reviewed.

Adams, R. L. P., 116

Adena, M. A., 1-12

Adhya, S. L. (Ed.), 242-243

Anand, R., 421

Arst, H. N., Jr., 129

Arthur, W., 335-346

Aung Thu, U., 126

Bailey, R. J., 1-12

Bainbridge, B., 418-419

Bayley, S. A., 122

Beale, G., 243-244

Berger, E., 13-23

Bhatt, B., 420-421

Bigger, T. R. L., 63-69

Bostock, C. J., 414-415

Boughey, H., 175-183

Boyce, A. J., 126

Broda, P., 122

Brown, A. H. D., 49-62

Bucheton, A., 357-369

Bukhari, A. I. (Ed.), 242-243

Burdon, R. H., 116

Burn, J., 424

Burnham, C. R. (Ed.), 109-110

Campbell, A. H., 116

Carothers, A. D., 127

Carter, C. O., 245-246

Garter, N. D., 419-420

Cartwright, R. A., 126

Cavelli-Sforza, L. L., 246

Chakraborty, R., 327-333

Chandley, A. C., 422

Chapelle, A. de la (Ed.), 116-118

Charlesworth, B., 137-153

Charlesworth, D., 137-153

Chater, K. F., 124

Clarke, B. F. C., 111-112

Cole, T. J., 399-402

Colson, C., 123-124

Connell, J. R., 423

Cook, P. J. L., 126

Coombes, D., 175-183

Cooper, A., 423

Cox, B. S., 121

Cullum, J., 122

Danford, N., 421

Dennis, E. S., 414

Douglas, S. D., 410-412

Dover, G., 415

Duggleby, C. J., 122
East, T. M., 347-356

Eaves, L. J., 249-320

Ebringer, A., 418-419, 419

Edwards, A. W. F., 125

Egel, R., 233-237

Emery, A. E. H., 421

Emes, E., 419-420

Eriksson, A. W., 124-125

Evans, H. J., 127

Evans, M. K., 105-107

Fannin, B., 122

Fennell, S. J., 413

Ferguson-Smith, M. A., 413

Firman, K., 122-123

Flavell, R., 413-414

Fletcher, J., 127, 422

Fowler, B., 423

Fraser, F. C. (Ed.), 409

Fudenberg, H. H., 410-412

Gallimore, P. H., 417

Gerlach, W. L., 414

Gibbons, D., 424

Gifford, E. M., Jr. (Ed.), 119

Glover, S. W., 122-123, 123

Gorton, D. J., 129

Green, M. M., 115-116

Gregson, N., 424

Halsey, A. H. (Ed.), 112

Harris, H. (Ed.), 409-410

Harris, R., 424

Heinaru, A. L., 122

Hennigan, E., 128

Herskowitz, I. H., 244-245

Hewitt, G. M., 347-356

Hill, W. G., 371-376

Hinata, K., 93-100

Hirschorn, K., 409-410

Holmes, R. S., 403-406

Houghton, J. A., 122, 421

Hughes, S. G., 124

Imbaraj, S., 424

Inouye, E. (Ed.), 110-111

Jadhav, M., 424

Jayasekara, N. E. M., 83-92

Jinks, J. L., 83-92, 175-183

Jones, R. N., 35-48 
Karlin, S., 165-174

Khokhar, M. T., 421

Killick, R. J., 215-225

King, R. C. (Ed.), 246-247

Kita, F., 377-383

Klouda, P. T., 424

Knowles, J., 243-244

Krystyna, A., 249-320

Kyu, Daw Hta, 126

La Cour, L. F., 101-103

Lamb, B. C., 418

Lambert, J., 122

Law, C. N., 185-191

Lawler, S. D., 416-417

Leamy, L., 25-34

Levan, G., 416

Li, W.-H. (Ed.), 239-242

Lovell, D. P., 418

Lycan, D., 128

Lyons, C. Y., 423

Mace, M., 420

McIntosh, R. A., 71-82

Malcolm, S., 413

Martin, D. E., 127

Martin, N. G., 249-320

Martinelli, S. D., 129

Maynard Smith, J., 205-214

Michaelis, A., $115-116$

Min, T., 42I

Mundy, C. R., 121

Murray, B. G., 227-231

Narayan, R. K. J., 415-416

Nei, Masatoshi, 118

Nevo, E., 49-62

Newton, M. E., 419

Ngwa Suh, N., 418-419

Nishimura, N. (Ed.), 110-111

Nishio, T., 93-100

O'Brien, P., 122

Ockey, C. H., 422-423

O'Dell, M., 413-414

O'Donald, P., 165-174

Ong, G., 128

Page, Catherine, 321-325

Parkin, B. H., 419-420

Pateman, J. A. (Ed.), 112-114

Peacock. W. J., 414

Permaul, R., 128

Persuad, T. V. N., 114-115

Philippides, D., 128

Phillips, R. L. (Ed.), 109-110

Pink, J. R. L., 410-412

Pooni, H. S., 83-92

Portini, P., 193-203

Price, D. A., 424
Rees, H., 1-12

Rendel, J. M., 105-107

Rieger, R., 115-116

Roberts, D. F., 244

Rost, T. L. (Ed.), 119

Rostron, J., 407

Salmasi, A.-M., 413

Sano, Y., 377-383

Sardharwalla, I. B., 423

Sassaman, C., 385-397

Scazzocchio, C., 128, 129

Scazzocchio, S., 128

Scott, D., 423

Sdrin, N., 128

Seabright, M., 424

Sealy-Lewis, H., 128

Seuanez, H., 127, 442

Shapiro, J. A. (Ed.), 242-243

Shufflebotham, W., 175-183

Sibert, J. R., 424

Sidebottam, E., 417

Smellie, R. M. S., 116

Smith, D., 413-414

Smith, J. E. (Ed.), 112-114

Sorsa, M. (Ed.), 116-118

Southern, D. I., 419

Stenseth, N. Chr., 205-214

Subramanian, R., 419

Sutherland, J., 13-23

Sutka, J., 185-191

Taylor, A. M. R., 422-423

Taylor, S., 128

Teoh, S. B., 35-48

Thompson, E. A., 125

Thomson, E., 422

Tuite, M. F., 121

Walker, P. M. B., 414

Wang, An-Chuan, 410-412

Waring, R. B., 129

Webb, G. C., 131-136

West, C. M., $419-420$

Westerman, M., 131-136

Williams, E., 122

Williamson, C. J., 215-225

Williamson, E., 420

Williamson, R., 413

Wilson, J. G. (Ed.), 409

Wood, R. H., 419

Worgan, D., 420

Worland, A. J., 185-191

Yasuda, N., 155-163

Yokoyama, S., 327-333

Young, P. A., 249-320

Zohary, D., 49-62 